\title{
A ROADMAP FOR AUTOSTEREOSCOPIC MULTI-VIEWER DOMESTIC TV DISPLAYS
}

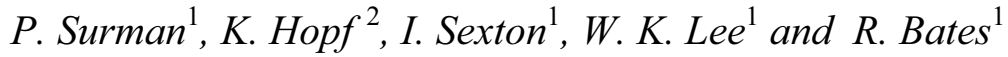 \\ ${ }^{1}$ De Montfort University, UK \\ ${ }^{2}$ Fraunhofer-Institute for Telecommunications - Heinrich-Hertz-Institut, Germany
}

\begin{abstract}
This paper presents a brief overview of the current technologies and technical approaches that may lead to viable and user-acceptable domestic autostereoscopic multiviewer television displays. It illustrates the performance attributes of the various technological approaches and points to the most likely approaches to succeed within the next 10 years. Finally, it shows possible timescales for the enabling technologies for 3D display, and concludes that multi-user autostereoscopic displays may be the first to gain widespread use.
\end{abstract}

\section{INTRODUCTION}

When considering the future of $3 \mathrm{D}$ displays it is essential to appreciate that this future is driven by user needs, not by potential solutions. In the case of 3D these needs are less straightforward than for example semiconductors, where Moore's law can be used to determine feature size and lower cost. It is unrealistic to look ahead further than ten years, as it is difficult to predict reliably beyond this. Also, this time scale fits with the anticipated introduction of 3D television systems. These are likely to be introduced within this period due to rapidly developing enabling technologies.

The future requirements of users in the area of 3D displays are not always entirely clear. For example there is no absolute consensus, even amongst $3 \mathrm{D}$ researchers, that consumers will prefer a 3D display over, say, a large high definition display. Also, although there is a general assumption that viewers will find the wearing of special glasses unacceptable, this seems to be based on anecdotal evidence only. However it is assumed in this paper that consumers will prefer the addition of 3D in many applications, and that it must be glasses free (autostereoscopic). It is currently not clear which basic 3D display technique will be adopted. The simplest way of presenting $3 \mathrm{D}$ is to supply binocular disparity only so that each eye sees a different image from separate viewpoints. Disparity is the most important cue and provides a strong 3D impression. However, presenting only disparity suffers from certain disadvantages. Other possible display types that could be considered are multi-view, holoform, volumetric and holographic. Another consideration is that of display size, which can range from wearable micro devices through to large scale theatre presentation. In addition, the display will not be the end of a stand-alone transmission system as has been the case with television in the past, but will be one of the interfaces of a converged system where the display could be any size from near-to-eye devices, through multi-user domestic TV-type displays, to theatre and cinema with a large number of viewers. 3D will probably more popular in some applications, for example in displays that have a viewing environment similar to today's television, and perhaps less popular in others.

\section{TECHNOLOGY REQUIREMENTS}

In order to determine technology requirements it is useful to summarise the results of the possible approaches. The generic types of 3D display are: binocular, multi-view, holoform, volumetric, and holographic and they are defined as follows:

\subsection{Binocular}

A binocular display is one where only two images are presented to the viewers. The viewing regions may occupy fixed positions, or may move to follow the viewers' head positions under the control of a head tracker.

\subsection{Multi-view}

In a multi-view display a series of discrete images are presented across the viewing field.

\subsection{Holoform}

A holoform display is defined as a multi-view display where the number of images presented is sufficiently large to give the appearance of continuous motion parallax and there is no difference between the accommodation and convergence of the viewers' eyes. Integral imaging can be considered as a type of holoform display where a large number of views are effectively produced from a high-resolution image in conjunction with a lenticular view-directing screen.

\subsection{Volumetric}

A volumetric display presents a $3 \mathrm{D}$ image within a volume

This work is supported by EC within FP6 under Grant 511568 with acronym 3DTV 
Table 1 Potential Autostereoscopic Display Performance

\begin{tabular}{|c|c|c|c|c|c|c|}
\hline \multicolumn{2}{|c|}{ Display type } & No. of viewers & $\begin{array}{c}\text { Viewer } \\
\text { movement }\end{array}$ & $\begin{array}{c}\text { Motion } \\
\text { parallax }\end{array}$ & $\begin{array}{c}\text { Acc./conv. } \\
\text { rivalry }\end{array}$ & $\begin{array}{c}\text { Image } \\
\text { transparency }\end{array}$ \\
\hline \multirow{3}{*}{ Binocular } & Fixed - non HT & Single & Very limited & No & Yes & No \\
\cline { 2 - 7 } & Single user HT & Single & Adequate & Possible & Yes & Yes \\
\cline { 2 - 7 } & Multi-user HT & Multiple & Large & Possible & Yes & No \\
\hline Multiple-view & Multiple & Limited & Yes & No & No \\
\hline Holoform & Multiple & Large & Yes & No & Yes \\
\hline
\end{tabular}

\subsection{Holographic}

The ideal stereoscopic display would produce images in real time that exhibit all of the characteristics of the original scene. This would require the reconstructed wavefront to be identical and could only be achieved using holographic techniques.

\subsection{Summary}

Table 1 summarises the performance of the various types, but does not take into account any other considerations, for example cost, complexity or whether the technology exists yet to support it.

\section{3D DISPLAY POSSIBILITIES}

\subsection{Motion parallax}

This property gives the ability to 'look around' an object by providing a changing image with changing viewpoint. Multi-view, and all types of binocular display, suffer from the disadvantage that the viewer's eyes focus on the screen, but converge at the apparent distance of the point in the image where the eyes are fixated. Any difference in the accommodation and convergence can potentially cause eyestrain and nausea [1]. Image transparency in volumetric displays is the effect where the front surface on an object appears transparent and allows objects behind this surface, which should be occluded, to be seen.

\subsection{Multiple view}

A large class of autostereoscopic displays can be usefully termed 'multiple view' (not to be confused with multi-view) and are defined as those where two or more different twodimensional views are presented across the viewing field. These range from binocular with two images, multi-view with between three and several hundred images, through to between multi-view and holoform is based on the work of several researchers who have determined the criteria for the appearance of smooth motion parallax and for the accommodation and convergence of the eyes to be the same. The Telecommunications Advancement Organisation (TAO) in Japan has identified that when the pupil receives two or more parallax images, the provision of a large number of views will allow the eye to focus at the same distance as the convergence [2]. They have produced a super multi-view display (SMV) based on this principle. It can be considered that the effect of the image appearing to 'jump' between adjacent views is similar to aliasing when a waveform is undersampled. A similar figure has been obtained by the Heinrich Hertz Institut who state that typically 20 views per interocular distance are required for the appearance of smooth motion parallax [3].

The range of multiple view displays is shown in Figure 1 where currently available displays appear at the lower end of the scale. Also shown are the numbers of views required to fulfil the TAO criterion over one metre and 2.5 metre field widths.

\subsection{Volumetric}

Although the current generation of volumetric displays are currently generally unsuitable for many 3D applications due to image transparency, it is possible that in the future displays with opaque voxels could be developed. The advantage volumetric displays have over multiple view displays is that each voxel need only be displayed once - as opposed to $\mathrm{N}$ times for an $\mathrm{N}$-view display. This gives these displays a greater efficacy in terms of display usage. They also do not give accommodation/convergence rivalry and provide motion parallax in both the horizontal and vertical directions. 
Multi-view

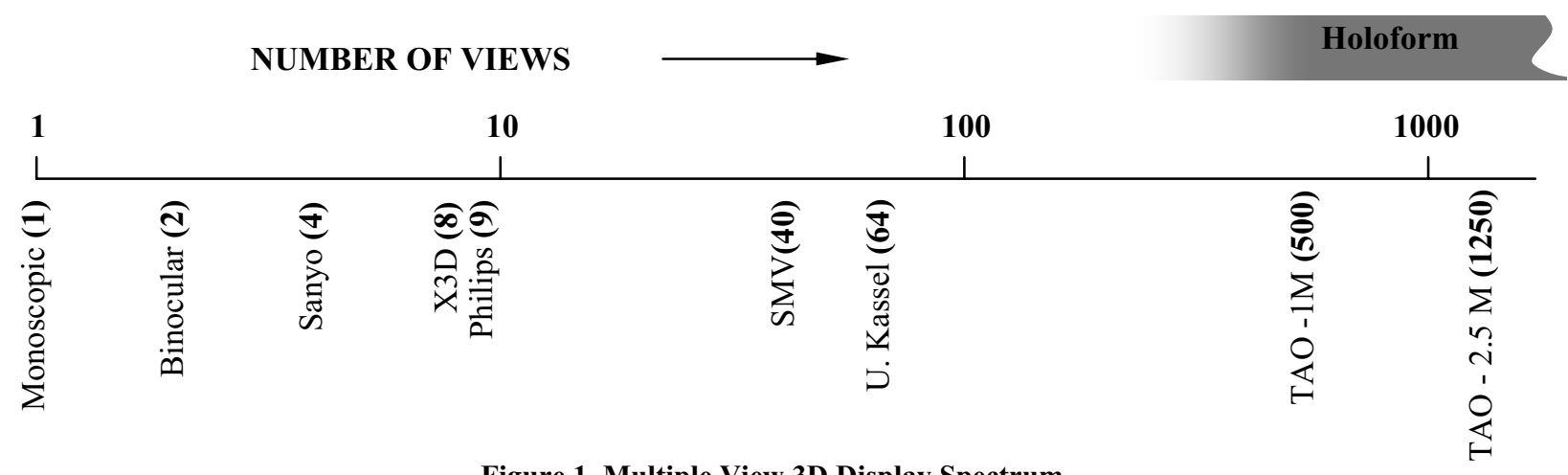

Figure 1. Multiple View 3D Display Spectrum

\subsection{Head Tracking}

A head tracking system can be used in order to make binocular displays more convenient to view by allowing a greater degree of viewer movement. Head tracking can be utilised in several ways. In a single user display it can enable the user to have a comfortable amount of movement. It can also be used in a multi-user display to supply the same pair of images to several viewers. This is the subject of work currently being carried out by the authors where a single direct-view LCD shows an image pair simultaneously on alternate pixel rows (spatial multiplexing). Novel backlighting under the control of a head tracker enables the images to be observed in the viewing field at the positions of the appropriate eyes. If the LCD can deliver $2 \mathrm{~N}$ views, either by spatial or temporal image multiplexing, motion parallax can be supplied to $\mathrm{N}$ viewers. Another application of head tracking could be to direct different images to different viewers, or to alter the image content for interactive purposes (for example to provide different views to different players for games applications).

\section{TOWARD 3D TECHNOLOGIES}

A useful method of determining the technology requirements is to consider each generic display type and then consider the requirements for each. This method has been chosen as it is not clear at the present time which, if any, generic type will dominate the market. It may well be that different types are preferred for different applications; for example, binocular displays may dominate the mobile market and holoform the multi-user domestic market

Table 2 shows a selection of enabling technologies that will be necessary for the implementation of a 3D TV display. The information is obtained from reports produced in 2006 by the European Union's 3D TV Network of Excellence.
The grey bar indicates the estimated date of introduction for each particular technology. The list of technologies is not exhaustive but it does represent those that will enable a wide variety of approaches to be implemented. Some of the technologies are already available and these are indicated by the grey bars covering the complete width, for example for LCDs that are used for fixed viewing zone non-head tracked binocular displays. This type of display is well established, with view directing screens, which include parallax barriers, prismatic screens and lenticular sheets, already optimised. This is also the case for the $2 \mathrm{D} / 3 \mathrm{D}$ switching layer. Although there may be incremental improvements in the near future, the technology is mature. One possible advance would be the adoption of different FPD technology, for example OLEDs. Similarly, head tracked single user displays are now available and are unlikely to change dramatically in the future. One type of this display, that enables good freedom of viewer movement, would benefit from the use of an LCD with its RGB sub-pixels running in the horizontal direction in order for the screen to be used in the landscape orientation.

A multi-user head tracked display that presents the same image pair to each viewer is under development by the authors. This utilises a non-intrusive head tracker to control a steering backlight unit of a direct-view LCD. Current LCDs are not particularly suitable for this purpose due to diffraction that scatters the light. A special LCD panel must be developed that has low diffraction, but also a good viewing angle. The function of this display could also be carried out with a scanned laser display used in conjunction with a spatial light modulator, a light valve and a specially designed superlens screen. This would be efficient as it would not be subject to the high light absorption losses of LCD displays. Multi-view displays are commercially available now and will the first multi-user autostereoscopic 
Table 2. Time Scales for Development of Principal Enabling Technologies

Developed Not yet developed

\begin{tabular}{|c|c|c|c|c|c|c|c|c|c|c|c|c|c|}
\hline \multicolumn{2}{|c|}{ Generic display type } & $\begin{array}{l}\text { Enabling } \\
\text { technology }\end{array}$ & ஓ્̊ & હે̊ & હ্ণ & હ્ડ & 륙 & 戸্ন & ָั & $\stackrel{\oplus}{\stackrel{\sim}{~}}$ & 弚 & $\stackrel{\text { ñ }}{\circ}$ & $\stackrel{0}{\stackrel{2}{*}}$ \\
\hline \multirow{14}{*}{ 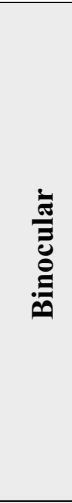 } & \multirow{4}{*}{$\begin{array}{c}\text { Fixed - non } \\
\text { head } \\
\text { tracked }\end{array}$} & LCD & & & & & & & & & & & \\
\hline & & OLED & & & & & & & & & & & \\
\hline & & View-directing screen & & & & & & & & & & & \\
\hline & & 2D/3D switching & & & & & & & & & & & \\
\hline & & & & & & & & & & & & & \\
\hline & \multirow{3}{*}{$\begin{array}{c}\text { Single user } \\
\text { head } \\
\text { tracked }\end{array}$} & Robust single-user HT & & & & & & & & & & & \\
\hline & & Horizontal pixel LCD & & & & & & & & & & & \\
\hline & & & & & & & & & & & & & \\
\hline & \multirow{6}{*}{$\begin{array}{l}\text { Multi-user } \\
\text { head } \\
\text { tracked }\end{array}$} & Multi-user HT & & & & & & & & & & & \\
\hline & & Steering backlight unit & & & & & & & & & & & \\
\hline & & Suitable LC panel (low diffraction) & & & & & & & & & & & \\
\hline & & RGB laser & & & & & & & & & & & \\
\hline & & Light valve & & & & & & & & & & & \\
\hline & & Suitable SLM & & & & & & & & & & & \\
\hline & & & & & & & & & & & & & \\
\hline \multirow{5}{*}{\multicolumn{2}{|c|}{ Multi-view }} & High-resolution LCDs $(<20 \mu \mathrm{m})$ & & & & & & & & & & & \\
\hline & & High-resolution OLED $(<20 \mu \mathrm{m})$ & & & & & & & & & & & \\
\hline & & Multi-view video projector & & & & & & & & & & & \\
\hline & & Direction selective screen & & & & & & & & & & & \\
\hline & & Multiple view rendering & & & & & & & & & & & \\
\hline & & & & & & & & & & & & & \\
\hline \multirow{4}{*}{\multicolumn{2}{|c|}{ Holoform }} & Very high res. OLED $(\sim 1 \mu \mathrm{m})$ & & & & & & & & & & & \\
\hline & & Very fast LV ( $>10^{4}$ FPS $)$ & & & & & & & & & & & \\
\hline & & Large $(>1 \mathrm{~m} \times .6 \mathrm{~m}) \mathrm{FE}$ barrier & & & & & & & & & & & \\
\hline & & Large $(>1 \mathrm{~m} \times .6 \mathrm{~m}) \mathrm{HOE}$ & & & & & & & & & & & \\
\hline \multirow{3}{*}{\multicolumn{2}{|c|}{ Volumetric }} & Multi-laver LC screen ( $>50$ lavers) & & & & & & & & & & & \\
\hline & & Very fast LV $\left(>10^{4} \mathrm{FPS}\right)$ & & & & & & & & & & & \\
\hline & & Voxel opacity & & & & & & & & & & & \\
\hline
\end{tabular}

display technology to be in widespread use. Although they have a limited available viewing region, they are of a simple construction and have a compact housing size. The displays will benefit from higher resolution displays in order to provide a greater number of views. OLEDs may also replace LCDs in this application. Large multi-view displays could also be realised with the use of projection in conjunction with a view-directing screen. Holoform displays that effectively display typically hundreds of images are a longer term prospect as they will involve the use of technology such as very high resolution displays, fast light valves, large ferro-electric parallax barriers and large holographic optical elements (HOEs). The anticipated time for this type of display to be developed is several years later than multiview or head tracked displays. Although volumetric displays have certain advantages, it is possible that a viable display that is compact and does not exhibit image transparency, may not be developed within the next ten years. Holography is not yet probable as it is extremely unlikely that a large screen holographic display will be developed within the period.

\section{CONCLUSIONS}

It is likely that with the advance of the enabling technologies viable and usable 3D domestic will be available and widespread within the next 10 years. Multiuser autostereoscopic display technology will likely pioneer roll-out, with holographic displays still some years away. This work has been carried out within the European Unionfunded Framework 6 '3D TV Network of Excellence' project.

\section{REFERENCES}

[1] P. A. HOWARTH, "Empirical Studies of Accommodation, Convergence and HMD Use", HosoBunka Foundation Symposium, Tokyo, 1996.

[2] Y. KAJIKI, H. Yoshikawa and T. Honda, "ThreeDimensional Display with Focused Light Array" SPIE Proceedings, "Practical Holography X", Vol.2652, pp 106116, 1996.

[3] S. PASTOOR, "Human Factors of 3DTV: An overview of Current research at Heinrich-Hertz-Institut Berlin", IEE Colloquium "Stereoscopic Television" No. 1992/173, p11/3, 1992. 